\title{
Prosthetic Rehabilitation of Velopharyngeal
} Insufficiency: Pharyngeal Obturator Prostheses with Different Retention Mechanisms

\author{
Suleyman Hakan Tuna \\ Gurel Pekkan ${ }^{\text {b }}$ \\ Hasan Onder Gumus ${ }^{c}$ \\ Alper Aktas ${ }^{d}$
}

\section{ABSTRACT}

Pharyngeal obturator prostheses restore the congenital or acquired defects of the soft palate and allow adequate closure of palatopharyngeal sphincter. Two patients with soft palate defect and subsequent velopharyngeal insufficiency were rehabilitated using pharyngeal obturator prostheses which had different retention mechanisms. Since it is necessary for swallowing and intelligible speech, the patients were examined in terms of adequate velopharyngeal closure after prosthetic treatment. The results were satisfying for both the patients and physicians. (Eur J Dent 2010;4:81-87)

Key words: Pharyngeal obturator; Velopharyngeal insufficiency; Hypernasality; Retention.

I a Assistant Professor, Department of Prosthodontics, Faculty of Dentistry, Suleyman Demirel University, Isparta, Turkiye.

b Assistant Professor, Department of Dentistry, Dumlupinar University, Kutahya, Turkiye.

c Assistant Professor, Department of Prosthodontics, Faculty of Dentistry, Erciyes University, Kayseri, Turkiye.

d Lecturer and Research Assistant, Department of Oral Surgery, Faculty of Dentistry, Hacettepe University, Ankara, Turkiye.

- Corresponding author: Dr. S. Hakan Tuna Suleyman Demirel Universitesi,

Diş Hekimligi Fakültesi, Protetik Diș Tedavisi AD Isparta, Turkiye.

Phone: +902462113234 Fax: +902462370607

E-mail: shtunadahotmail.com

\section{INTRODUCTION}

The soft palate acts as a dynamic separator between oral and nasal cavity. ${ }^{1}$ The soft palate, lateral and posterior pharyngeal walls form the velopharyngeal (VP) closure so that all of them create a three dimensional muscular valve which is known as VP sphincter. ${ }^{2}$ The VP closure pattern depends on the contraction degree of the sphincter components. Variation in the contribution of each of these components produces several patterns of sphincteric closure or attempted closure of the VP portal that were described by Skolnick et al. ${ }^{2}$ Adequate VP closure is required during swallowing and production of all consonants except for the nasal ones. ${ }^{3}$ 
Impairment of VP function can be due to insufficiency or incompetency. ${ }^{3-8} \mathrm{VP}$ insufficiency is distinguished by speech and nasal resonance abnormalities related to defects of the soft palate, which may be congenital as in cleft lip and palate (CLP) or acquired as in palatal tumor resection..$^{5-7}$ VP incompetence describes dysfunction of an anatomically intact VP mechanism as in patients with neuromuscular disorders. ${ }^{3,4,8}$ The diagnosis of VP insufficiency can be made with perceptual speech evaluation, multiview videofluoroscopy (MVF), and nasendoscopy (NE). ${ }^{9}$

The primary effects of the VP insufficiency are air-flow escape and hypernasality. ${ }^{10,11}$ Secondary effects are disorders in speech articulation (distortions, substitutions and omissions). ${ }^{6,10} \mathrm{VP}$ insufficiency causes communication problems because of distortion in speech, resonance and articulation apart from swallowing disturbance. ${ }^{10-12}$ In this regard, patients usually have psychological problems together with physical difficulties. ${ }^{13}$ These problems are demonstrated especially in childhood and continue lifelong.

Surgery in combination with speech therapy is a common approach to the treatment of VP dysfunction. ${ }^{6,11}$ There are several surgical procedures that can be performed to correct the physical malfunction. Some of these are palatal pushback with a pharyngeal flap lining, sphincter pharyngoplasty, a superiorly based obturating pharyngeal flap, and Furlow palatoplasty. ${ }^{9}$ However, when surgical treatment is not considered as an option, prosthetic management of VP insufficiency is carried out by means of a pharyngeal obturator, whereas VP incompetence is traditionally managed by a palatal lift prosthesis. ${ }^{4,5,7,8} \mathrm{~A}$ pharyngeal obturator is a removable maxillary prosthesis which has a posterior extension to separate oropharynx and nasopharynx. ${ }^{5,10}$ This obturator prosthesis restores the defects of the soft palate and allows adequate closure of palatopharyngeal sphinter. ${ }^{5,7}$ When a pharyngeal obturator is placed, the patient can exhibit adequate separation between the oral and nasal cavities during production of plosives consonants or while blowing with variable intensity. ${ }^{14,15}$ An effective prosthesis will restore speech, allow proper swallowing, and have an acceptable appearance. ${ }^{16}$ However, it should have sufficient retention and stability. ${ }^{17-20}$ In dentulous and partially edentulous patients the retention and stability of the pharyngeal obturator prosthesis is easily achieved by the existing teeth. ${ }^{10,18} \mathrm{How}$ ever, it may be hard to achieve adequate retention with conventional prostheses in edentulous patients. 1,10,13,20 If dental implants are considered, implant-supported overdentures are the best and sometimes unique choice for treatment in such patients. ${ }^{7,17,19-21}$

Herein, we report two cases with soft palate defects rehabilitated by pharyngeal obturator prostheses using different retention mechanisms. Adequate VP closure was obtained with applied prosthetic treatments. Thereby, oro-nasal communication was prevented, esthetics and speech were improved.

\section{CASE 1}

A twenty four year-old female teacher suffering from speech difficulty and communication problems had a history of a cleft of the hard and soft palate. In her medical history, the patient had an operation for restoring the cleft palate during her early childhood. She had a maxillary anterior fixed dental prosthesis extending from the left first premolar to right canine (Figure 1). She was talking with soft intensity (ie, volume) to decrease the nasal emissions. Speech difficulty and hypernasality were also detected in the clinical examination. When the patient was instructed to say 'ah' a tethered velopharynx and insufficient velar elevation were observed due to the surgical scar at the velum. Speech evaluation was performed by speech pathologists that assessed resonance, the occurrence of inappropriate nasal air emission, and articulation. Nasal emission was assessed using the Bzoch base-10 index of Nasal Air Emission. Hypernasality scores were derived similarly on a base-10 index using the Bzoch Cul-de-Sac Test. Articulation was assessed using the Bzoch Error Pattern Articulation Test. ${ }^{22}$

The patient refused to have a surgical reconstruction. A pharyngeal obturator with clasp retention was planned since the patient had full dentition. The impression of the pharyngeal bulb (speech bulb or obturator) was made with yellow modeling plastic impression compound (Kerr Corp, Orange, California, USA) during the functional movements of the patient. The patient was asked to move her head in a circular manner from side to side, to extend her head as far forward and 
backward as possible and to say 'ah' and swallow. Afterwards, detailed impression was made with zinc-oxide eugenol impression material (SS White Impression Paste, Gloucester, England)..$^{14}$ The acrylic bulb was formed in wax and fabricated according to the impression using heat polymerized acrylic resin (Meliodent; Heraeus Kulzer GmbH, Hanau, Germanyl and prosthesis was delivered to the patient (Figures 2 and 3). The patient was instructed in the specific care for her prosthesis.

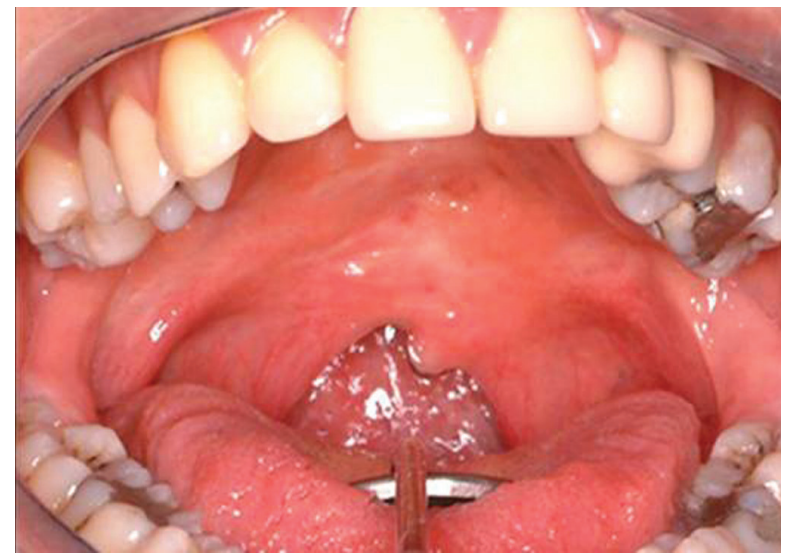

Figure 1. Intraoral view of the patient with congenital soft palate defect.

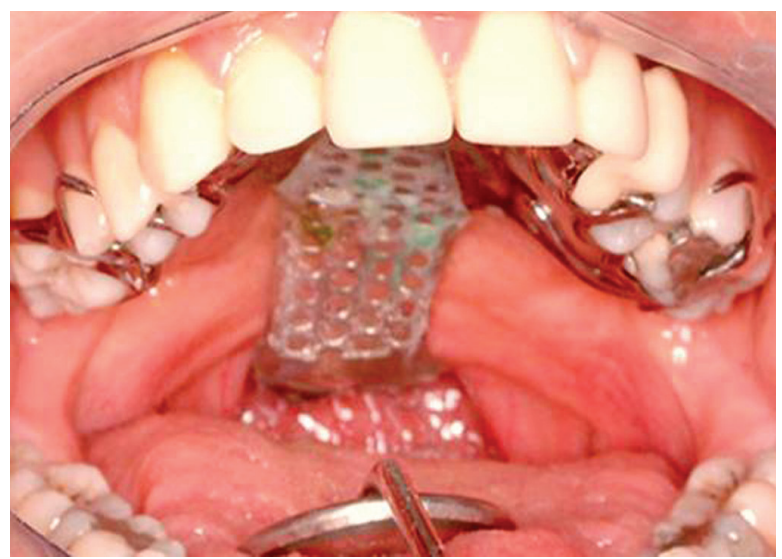

Figure 3. Intraoral view of the dentate patient's pharyngeal obturator.

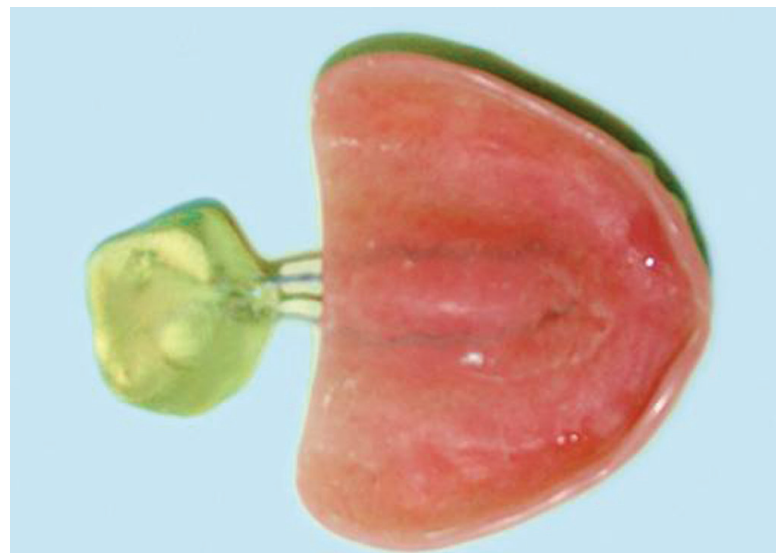

Figure 5. Maxillary complete denture with pharyngeal obturator.
After prosthetic rehabilitation, the speech of the patient was improved markedly. The speech improvement was confirmed by a speech pathologist so that the patient's facial grimaces were disappeared and articulation of the explosive consonants such as "p", "b", "g", "t", and "d" was corrected. During two years of follow-up period, patient was satisfied with the use of the prosthesis and also expressed her social and psychological satisfaction.

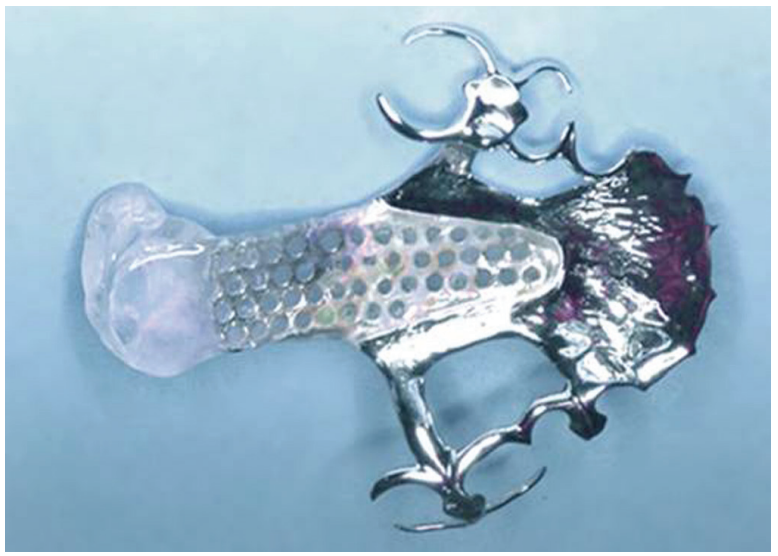

Figure 2. Pharyngeal obturator with clasp retention.

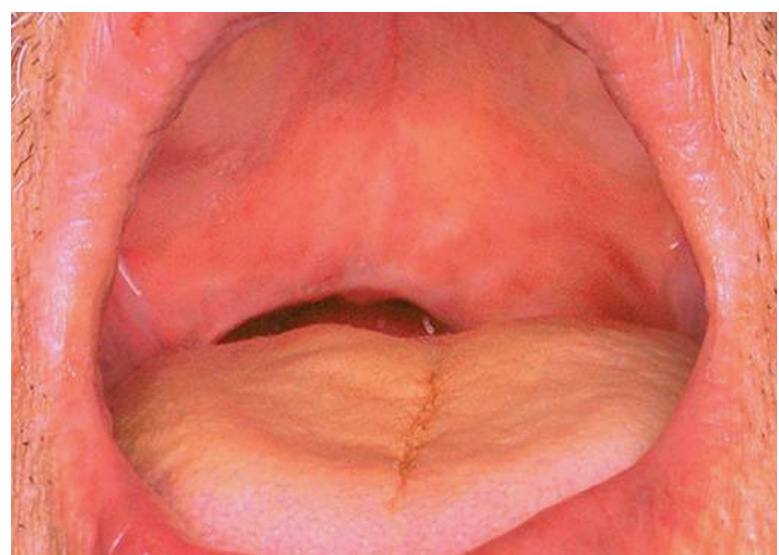

Figure 4. Intraoral view of the edentulous patient with aquired soft palate defect.

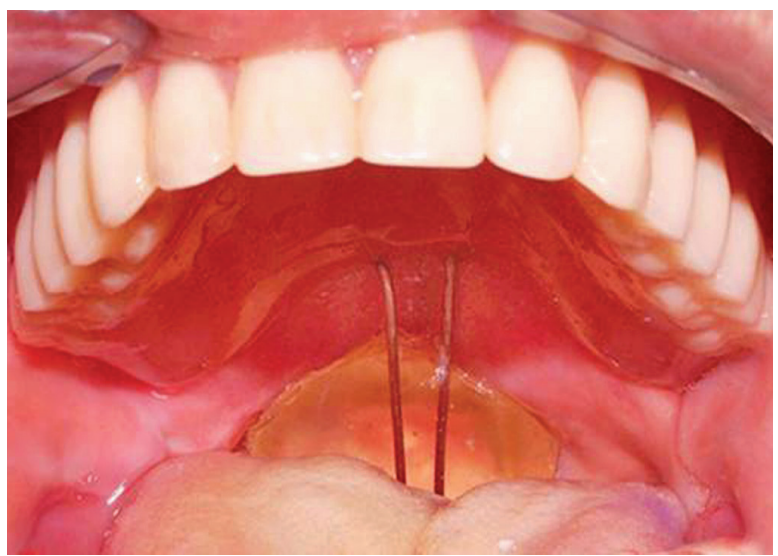

Figure 6. Intraoral view of the edentulous patient's pharyngeal obturator. 


\section{CASE 2}

A sixty four year-old completely edentulous male patient with VP insufficiency was referred with the diagnosis of soft palate defect (Figure 4). He had speech difficulty and hypernasality. A speech pathologist performed his speech evaluation including assessment of resonance, the occurrence of inappropriate nasal air emission, and articulation. His medical history revealed that he had an operation for squamous cell carcinoma of the soft palate one year prior to referral. Right lateral neck dissection was performed to him. Intraoral examination revealed that the alveolar ridges, vestibular sulci and shape of the palate were suitable for a conventional complete denture. He refused dental implant treatment, which was proposed for increasing the stability and retention of the prostheses.

Complete denture prostheses were fabricated for him, conventionally. Thereafter, functional impression of the soft palate defect area was made using green modeling plastic impression compound (Kerr Corp, Orange) with the support of the $1 \mathrm{~mm}$ round orthodontic wire (Dentaurum, Ispringen, Germanyl which was attached to the posterior part of the upper prosthesis. Final impression was made using zinc oxide eugenol impression material (SS White Impression Paste, Gloucester) after instructing the patient for functional movements of his head as in case 1. The acrylic pharyngeal bulb was processed from heat polymerizing acrylic resin (Meliodent, Heraeus Kulzer $\mathrm{GmbH}$ ). Definitive prostheses were delivered to the patient after making necessary adjustments (Figures 5 and 6).

Adequate VP closure was detected after the patient was examined during drinking water in the upward head position. Moreover, no nasal reflux was observed. A speech pathologist confirmed that the hypernasality was reduced after testing the production of oral and nasal consonants and the speech was noticeably improved after perceptual speech evaluation. The patient was trained in oral hygiene and instructed in the specific care for his new prostheses. The checkups were done at $1^{\text {st }}$ week, $2^{\text {nd }}$ week, 1 month, and 6 months after insertion of the prostheses. After three years follow-up period, the patient was satisfied with his prostheses.

\section{DISCUSSION}

Prosthetic rehabilitation of the patients suffering from VP deficits with obturator prostheses varies according to the location and nature of the defect or deficiency. ${ }_{4,5,7,8}$ There are differences between obturator prostheses constructed for patients with developmental or congenital malformations of the soft palate, as compared with those constructed for patients with acquired defects. $4,7,8,10$ However, the objectives of obturation are to provide the capability for the control of nasal emission and inappropriate nasal resonance during speech and to prevent the leakage of material into the nasal passage during deglutition. ${ }^{5,10,11}$ To achieve normal speech with a prosthesis, an accurate prognosis is extremely important for the patients exhibiting considerable movement of the residual VP complex during function. 2,11 Because the movement of the lateral pharyngeal walls is essential for the control of nasal emission, little or no movement of VP mechanism makes is difficult to achieve normal speech with either surgical re-

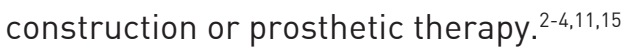

In the literature, several types of prostheses have been described to improve speech ability. $4,5,7,11,18,21,23$ A pharyngeal obturator prostheses may prevent the hypernasality and/or nasal emission associated with VP inadequacies. ${ }^{4,5}$ In order to obtain adequate VP closure during speech and swallowing a posterior extension is added to prosthesis. ${ }^{5,14}$ The extension must be positioned at the level of the hard palate during the most active movement of the pharyngeal sphincter. ${ }^{10,15}$ This movement can be achieved by asking the patient to say 'ahh' or by touching to posterior wall of the pharynx with an instrument to initiate gag reflex. ${ }^{10,14}$ An acrylic resin extension must be formed functionally. This extension must be in static contact with the soft tissues and must not affect the stability of the prosthesis., 10 The impression should be examined for contact with the pharynx bilaterally and posteriorly. ${ }^{14}$ In this report, patients were allowed to drink water to test the complete closure of the anatomical defect of soft and hard palate. The water should not reflux into the nasal cavity when the patient is in upright position. ${ }^{10}$

The success of the soft palate defect prosthesis depends on the functional adaptation of the impression material. ${ }^{7,10,14}$ In current cases, modeling plastic impression compound was used in 
functional contouring of the palatal defect and VP portion. Zinc oxide impression material was added to make the final impression. Zinc oxide-eugenol impression material is accurate with dimensional changes during setting of about $0.1 \% .^{14}$ The mixed materials have adequate adhesion to compound; therefore, adhesives aren't necessary to be applied to the compound in clinical usage. Moreover, no separating medium is needed before the stone model is poured. However, it sets to a brittle solid and needs to be handled carefully. Life-threatening complications may arise in the case of broken and aspirated zinc oxide-eugenol impression material. ${ }^{14}$ Rarely some patients develop eugenol irritation in the soft tissues. Impression wax may also be added to the compound surface to make final impression. ${ }^{17}$ Recently, elastomeric impression materials such as polyvinylsiloxane and polyether have also been considered appropriate for border molding procedures. ${ }^{24}$

Retention of pharyngeal obturator can be obtained by direct and indirect retainers for patients with complete maxillary dentition, as in the first case. Although removable partial denture designs for patients with VP deficiencies are similar to removable partial denture designs for nonsurgical patients, the long lever arm created by the exten-

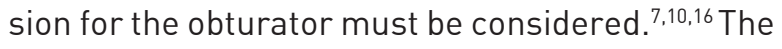
weight and length of obturator portion increases the effect of gravitational forces and the potential for rotation around the fulcrum line. The effect of extension will be most significant for patients requiring a Kennedy Class I or Class II removable partial denture and minimal for patients with Class III or Class IV removable partial dentures..$^{10}$ For patients requiring Class I or Class II partial dentures, multiple indirect retainers are suggested, which will tend to resist the downward displacement of the obturator and increase the stability of the prosthesis. ${ }^{25}$

In edentulous patients, achieving an effective retention by conventional prostheses for the edentulous patients with both hard and soft palate defects is very difficult, if not impossible. $7,19,20$ This is especially due to the weight of the prosthesis and the inability to obtain a border seal. ${ }^{17}$ Moreover, a complete denture may be contraindicated in the patients with irregular palate anatomy, shallow vestibular sulci or lower muscle attachments. ${ }^{16}$ Therefore, dental implants have great importance for these patients. ${ }^{21}$ Accordingly, the retention provided by the implants will be enhanced if the implants can be positioned to maximize the anterior-posterior spread. They improve prosthesis retention, stability, and occlusal function when they are used in selected cases. ${ }^{21}$ However in some cases implant treatment may be complicated such as for the irradiated tissues. ${ }^{26}$ Furthermore implant treatment may be rejected by the patients because of psychological reasons as presented in second case report. The retention of prosthesis can be obtained by the alveolar ridge and deep vestibular sulcus as in the second case in this report.

The degree of defect affects the functions of the obturator. If the defect includes both soft and hard palate resections, the discomfort in the usage of obturator increases. ${ }^{27}$ Most individuals with a history of radiation therapy have poorer satisfaction with obturator function due to their dry mouth. ${ }^{27}$

The treatment of VP insufficiency requires multidisciplinary approach. Accordingly, a speech pathologist should participate in treatment of these cases to test articulation errors and inappropriate oro-nasal resonance balance. ${ }^{7}$ Perceptual and instrumental measures of hypernasality and nasal escape along with a profile of the patient's articulation provide the diagnostician information about the frequency and consistency of VP insufficiency. These measures, however, provide only limited information about the functioning of the VP mechanism. The use of MVF and/or NE may contribute to the diagnostic confirmation of the assessment of velar mobility, pattern of velar elevation, size of residual VP gap and lateral pharyngeal wall displacement while the patient is producing a standardized sample of connected speech. It may also contribute the assessment of treated patients with VP insufficiency. ${ }^{9,28}$ While neither NE nor MVF can substitute for perceptual speech assessment in the diagnosis of VPI, they are complementary tools in the assessment of velopharyngeal function. ${ }^{28}$ In present cases, no nasopharyngoscopic evaluations were made. However, perceptual speech evaluations were demonstrated significant improvements in speech ability and VP function.

In the course of time dissatisfaction of patients using definitive obturator increases. It seems that adjustment to an obturator might be a lengthy and changing process that requires close clinical monitoring. ${ }^{27}$ 


\section{CONCLUSIONS}

In this report, two patients with soft palate defect as VP insufficiency and different oral conditions were treated successfully by pharyngeal obturator prostheses which had different retention mechanisms. It is crucial to rehabilitate these patients with suitable prosthetic management for successful results.

\section{REFERENCES}

1. Zarb GA, Blonder CL. Prosthodontic Treatment for Edentulous Patient: Complete Dentures and Implant-Supported Prostheses. In: Jacob RF. Maxillofacial prosthodontics for the edentulous patient. St. Louis: Mosby Inc; 2004. p. 449470.

2. Skolnick L, McCall GN, Barnes M, The sphincteric mechanism of velopharyngeal closure. Cleft Palate J 1973;10:286305.

3. Johns DF, Rohrich RJ, Awada M. Velopharyngeal incompetence: a guide for clinical evaluation. Plast Reconstr Surg $J$ 2003; 112:1890-1897.

4. Wolfaardt JF, Wilson FB, Rochet A, McPhee L. An appliance based approach to the management of palatopharyngeal incompetency: A clinical pilot project. J Prosthet Dent 1993;69:186-195.

5. Saunders TR, Oliver NA. A speech-aid prosthesis for anterior maxillary implant-supported prostheses. J Prosthet Dent 1993;70:546-547.

6. Ragab A. Cerclage sphincter pharyngoplasty: a new technique for velopharyngeal insufficiency. Int J Pediatr Otorhinolaryngol 2007;71:793-800.

7. Abreu A, Levy D, Rodriguez E, Rivera I. Oral rehabilitation of a patient with complete unilateral cleft lip and palate using an implant-retained speech-aid prosthesis: Clinical report. Cleft Palate Craniofac J 2007;44:673-677.

8. Shifman A, Finkelstein Y, Nachmani A, Ophir D. Speech-aid prostheses for neurogenic velopharyngeal incompetence. J Prosthet Dent 2000;83:99-106.

9. Seagle MB, Mazaheri MK, Dixon-Wood VL, Williams WN. Evaluation and treatment of velopharyngeal insufficiency: the University of Florida experience. Ann Plast Surg 2002;48:464-470.

10. Beumer III J, Curtis TA, Marunick MT. Maxillofacial Rehabilitation: Prosthodontic and Surgical Considerations; Speech, Velopharyngeal Function, and Restoration of Soft Palate Defects. St. Louis: Ishiyaku EuroAmerica, Inc; 1996. p. 285-324.

11. Yoshida H, Michi K, Yamashita Y, Ohno K. A comparison of surgical and prosthetic treatment for speech disorders attributable to surgically acquired soft palate defects. J Oral Maxillofacial Surg 1993;51:361-365.
12. Werkmeister R, Szulczewski D, Walteros-Benz P, Joos UJ. Rehabilitation with dental implants of oral cancer patients. J Craniomaxillofac Surg 1999;27:38-41.

13. Hickey AJ, Salter M. Prosthodontic and psychological factors in treating patients with congenital and craniofacial defects. J Prosthet Dent 2006;95:392-396.

14. Keyf F, Sahin N, Aslan Y. Alternative impression technique for a speech-aid prosthesis. Cleft Palate Craniofac $J$ 2003;40:566-568.

15. Tachimura T, Nohara K, Wada T. Effect of placement of a speech appliance on levator veli palatini muscle activity during speech. Cleft Palate Craniofac J 2000;37:478-482.

16. Branemark P-I, Higuchi KW, Oliveira de MF. Rehabilitation of complex cleft palate and craniomaxillofacial defects: the challenge of Bauru. Illinois: Quintessence; 1999. p. 17-30.

17. Harrison JW. Dental implants to rehabilitate a patient with an unrepaired complete cleft of the hard and soft palate: a clinical report. Cleft Palate Craniofac J 1992;29:485-488.

18. De Carvalho WR, Barboza EP, Caula AL. Implant-retained removable prosthesis with ball attachments in partially edentulous maxilla. Implant Dent 2001;10:280-284.

19. Lund TW, Wade M. Use of osseointegrated implants to support a maxillary denture for a patient with repaired cleft lip and palate. Cleft Palate Craniofac J 1993;30:418-420.

20. Lefkove MD, Matheny B, Silverstein L. Implant prosthodontic procedures for a completely edentulous patient with cleft palate. J Oral Implantol 1994;20:82-87.

21. Tuna SH, Pekkan G, Buyukgural B. Rehabilitation of an edentulous cleft lip and palate patient with a soft palate defect using a bar-retained, implant-supported speechaid prosthesis: a clinical report. Cleft Palate Craniofac $J$ 2009;46:97-102

22. Bzoch KR. Measurement and assessment of categorical aspects of cleft palate language, voice and speech disorders. In: Bzoch KR, ed. Communicative disorders related to cleft lip and palate. 3rd ed. Boston: Little, Brown, 1989. p.137.

23. Leonard TJ, Gillis R. Differential effects of speech prostheses in glossectomized patients. J Prosthet Dent 1990;64:701708.

24. Petrie CS, Walker MP, Williams K. A survey of U.S. prosthodontists and dental schools on the current materials and methods for final impressions for complete denture prosthodontics. J Prosthodont 2005;14:253-262.

25. Jacob RF, King G. Indirect retainers in soft palate obturator design. J Prosthet Dent 1990;63:311-315.

26. Harrison JS, Stratemann S, Redding SW. Dental implants for patients who have had radiation treatment for head and neck cancer. Spec Care Dentist 2003;23:223-229. 
27. Rieger JM, Wolfaardt JF, Jha N, Seikaly H. Maxillary obturators: the relationship between patient satisfaction and speech outcome. Head Neck 2003;25:895-903.

28. Lam DJ, Starr JR, Perkins JA, Lewis CW, Eblen LE, Dunlap J, Sie KC. A comparison of nasendoscopy and multiview videofluoroscopy in assessing velopharyngeal insufficiency. Otolaryngol Head Neck Surg 2006;134:394-402. 